PHYSICAL REVIEW D 96, 099902(E) (2017)

\title{
Erratum: Dark photons from captured inelastic dark matter annihilation: Charged particle signatures [Phys. Rev. D 95, 075015 (2017)]
}

\author{
Jordan Smolinsky \\ Philip Tanedo \\ (Received 9 October 2017; published 6 November 2017)
}

DOI: 10.1103/PhysRevD.96.099902

The code used to produce the final results and plots in this paper contained a typographical error in the dark photon decay length. None of the equations in the paper needs to be modified, but Figs. 3-5 should be replaced by the updated versions shown below.
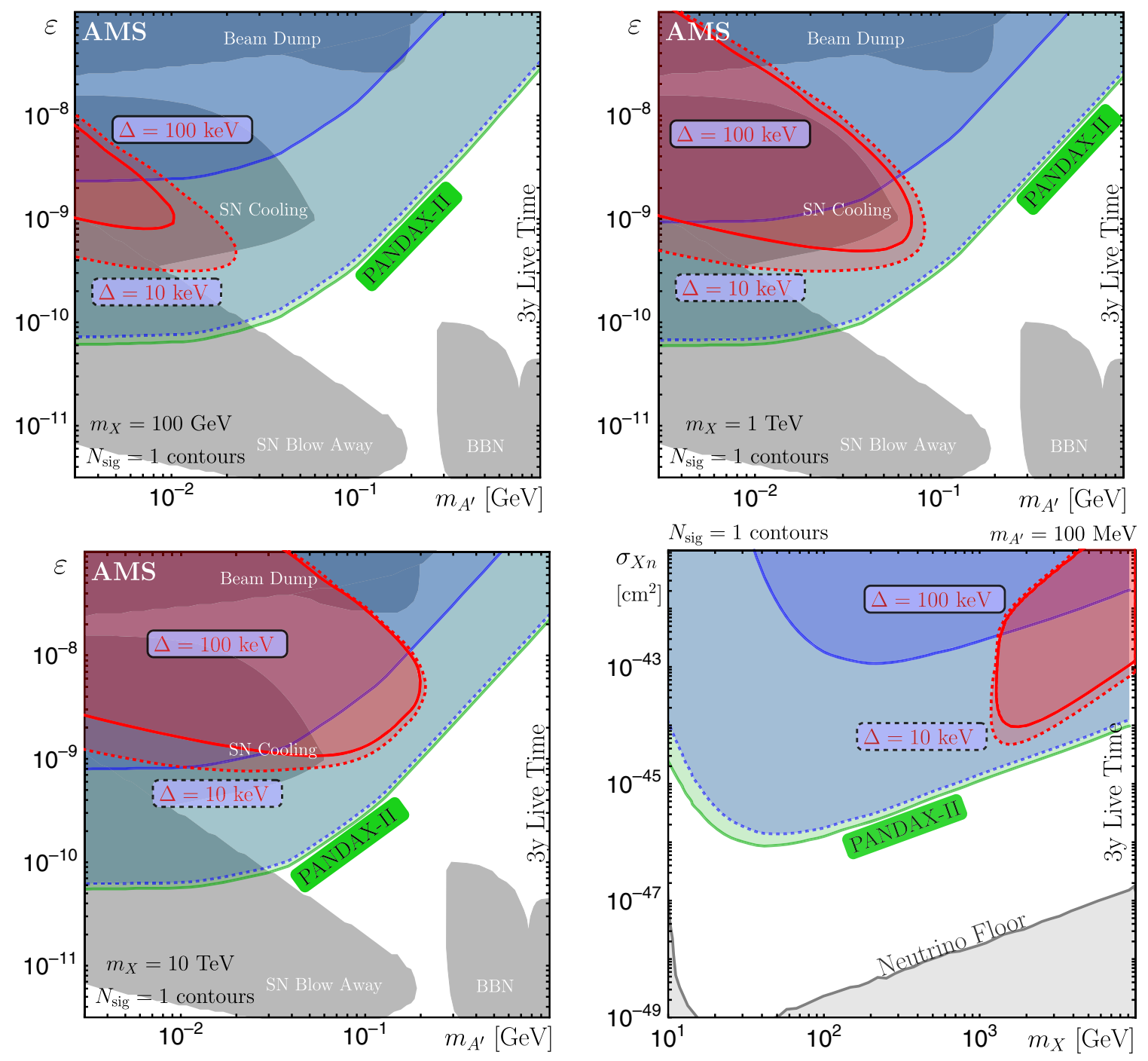

FIG. 3. Red: AMS-02 reach region for $T=3$ years live time in the $\left(m_{A^{\prime}}, \varepsilon\right)$ plane for $m_{X}=100 \mathrm{GeV}$ (top left), $m_{X}=1 \mathrm{TeV}$ (top right), and $m_{X}=10 \mathrm{TeV}$ (bottom left) and in the $\left(m_{X}, \sigma_{X n}\right)$ plane for $m_{A^{\prime}}=100 \mathrm{MeV}$ (bottom right). The dark sector fine-structure constant $\alpha_{X}$ is set by requiring $\Omega_{X} \simeq 0.23$. Solid curves are for the dark sector mass splitting $\Delta=100 \mathrm{keV}$, while dashed curves are for $\Delta=10 \mathrm{keV}$. The indirect detection reach is also compared to other probes. Green: current bounds from direct detection with $\Delta=0$ [1,2]. Blue: rescaled bounds from direct detection. Solid curves are for $\Delta=100 \mathrm{keV}$, while dashed curves are for $\Delta=10 \mathrm{keV}$. Gray: regions probed by beam dump experiments, supernova observations, and big bang nucleosynthesis constraints [3,4] (top and bottom left) and the neutrino floor (bottom right). 

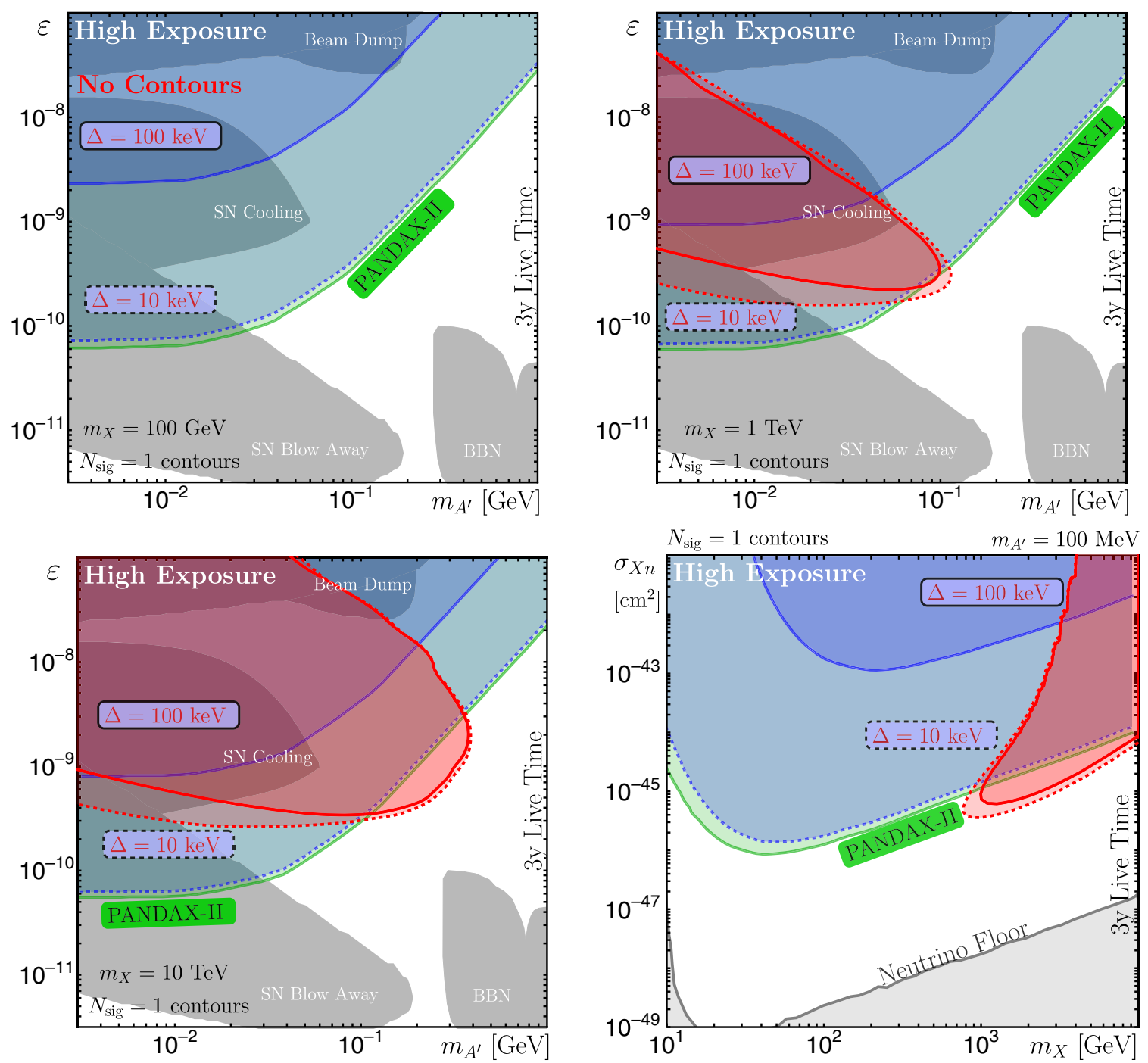

FIG. 4. Same as Fig. 1 but with increased exposure $\xi_{\odot}^{\text {high }}=80 \xi_{\odot}$. Note that the reach for $m_{X}=100 \mathrm{GeV}$ dark matter vanishes at AMS-02. This is because our angular and energy cuts, set by the condition that we expect only one background event, restrict the amount of signal positrons to be negligibly small. The positron background drops off at higher energies, so that a search for more massive dark matter does not suffer the same reduction in the expected signal rate.

In accordance with the modified results of other papers in the sequence, the region of parameter space described in the Introduction should be adjusted to read, "We discovered further that the same process occurring in the Sun, which we term 'dark sunshine,' may result in energetic positrons detectable by the Alpha Magnetic Spectrometer (AMS-02), providing a probe of the dark sector unconstrained by dark photon exclusions from fixed target experiments and supernova observations, with $100 \mathrm{GeV} \lesssim m_{X} \lesssim 10 \mathrm{TeV}, 1 \mathrm{MeV} \lesssim m_{A^{\prime}} \lesssim 100 \mathrm{MeV}$, and $10^{-10} \lesssim \varepsilon \lesssim 10^{-8}$." Similarly, the region of parameter space described in the Conclusions should read, "This leaves a region of parameter space, $1 \mathrm{TeV} \lesssim m_{X} \lesssim 10 \mathrm{TeV}, \Delta \sim 100 \mathrm{keV}, 10 \mathrm{MeV} \lesssim m_{A^{\prime}} \lesssim 100 \mathrm{MeV}$, and $10^{-10} \lesssim \varepsilon \lesssim 10^{-8}$, that is unprobed by supernova observations and fixed target dark photon searches and favored to resolve small scale structure problems, where an inelastic dark sector may still be discovered or excluded using existing experiments and data."

We are grateful to Adam Green for bringing the error to our attention. 

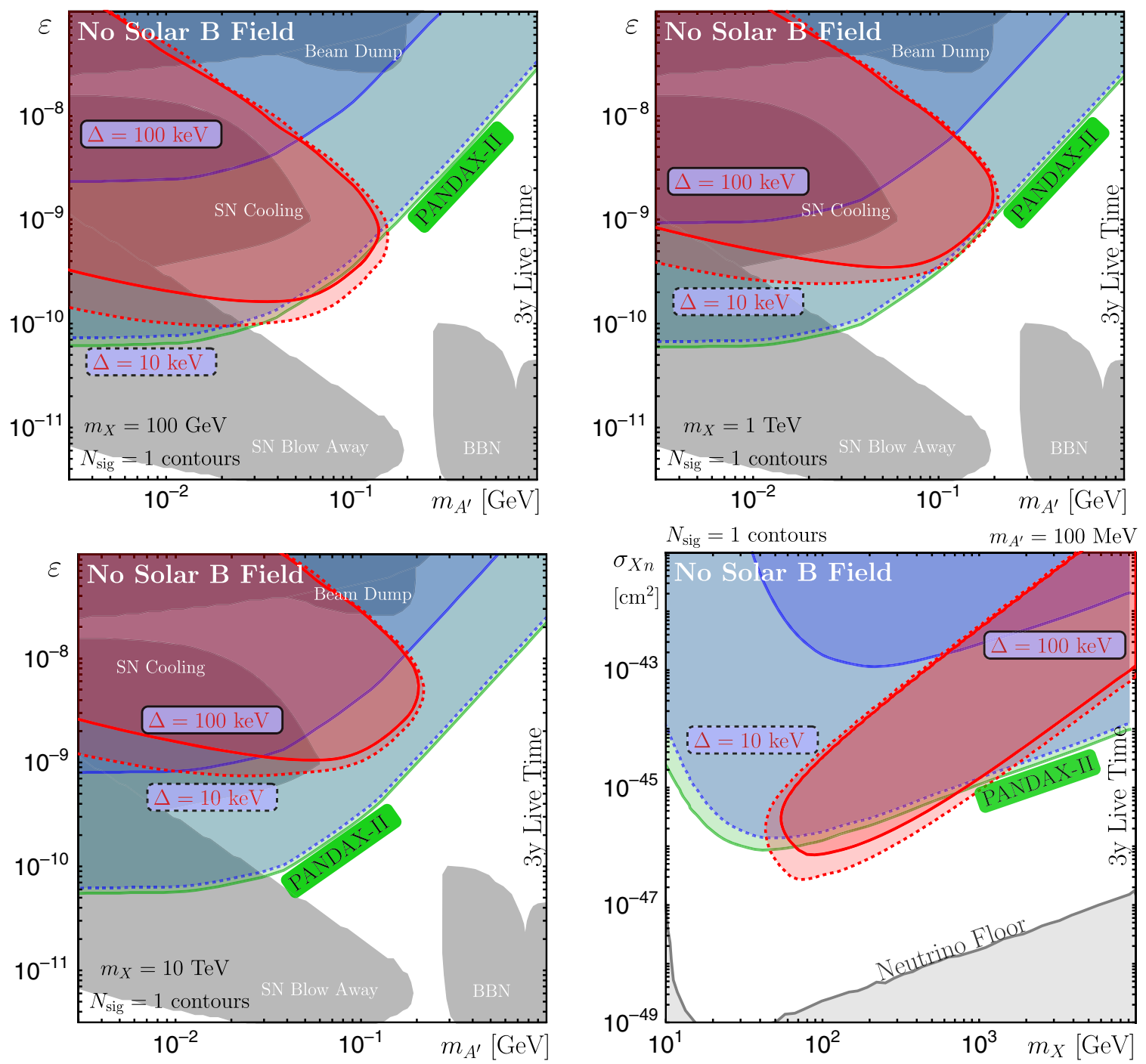

FIG. 5. Same as Figs. 1 and 2 but using the naive flux based estimate in which magnetic deflections are ignored.

[1] E. Del Nobile, M. Kaplinghat, and H.-B. Yu, Direct detection signatures of self-interacting dark matter with a light mediator, J. Cosmol. Astropart. Phys. 10 (2015) 055.

[2] X. Cui et al. (PANDAX-II Collaboration), Dark Matter Results From 54-Ton-Day Exposure of PandaX-II Experiment, arXiv:1708.06917.

[3] R. Essig et al., in Community Summer Study 2013: Snowmass on the Mississippi (CSS2013) Minneapolis, MN, 2013 (unpublished), arXiv:1311.0029.

[4] A. Fradette, M. Pospelov, J. Pradler, and A. Ritz, Cosmological constraints on very dark photons, Phys. Rev. D 90, 035022 (2014). 